into two groups-adjuvant pelvic radiation (116 patients) and no adjuvant treatment (114 patients). The propensity scores were employed to balance the 2 study groups with respect to distribution of confounding factors. The primary outcomes were disease-free survival (DFS) and overall survival (OS). The secondary outcomes included treatment complications and quality of life.

Results: The DFS were comparable between the groups with the adjusted hazard ratio (aHR) 0.46 (95\% confidence interval $[\mathrm{CI}]=0.18-1.16 ; \mathrm{p}=0.1)$ for adjuvant radiation. Similarly, the OS were not significantly different with the aHR 1.75 (95\% $\mathrm{CI}=0.63-4.90 ; \mathrm{p}=0.3$ ) for adjuvant radiation. Serious side effects were reported in 4 patients, 3 in the adjuvant radiation group ( 2 lymphedema and 1 enterovesical fistula) and 1 in the no adjuvant treatment group (lymphedema). Quality of life scores were not significantly different.

Conclusion: The survival benefit of adjuvant pelvic radiation for intermediate-risk early-stage cervical cancer could not be demonstrated in this study.

Oral (OC5)

Cervical Cancer

https://doi.org/10.3802/jgo.2021.32.S1.0C5

\section{Therapeutic role of conization before radical hysterectomy in patients with early cervical cancer}

\section{Ji Song Min, ${ }^{1}$ Ki Hyeon Song, ${ }^{2}$ Chel Hun Choi,, Tae-Joong Kim, ${ }^{1}$ Jeong-Won Lee, ${ }^{1}$ Yoo-Young Lee ${ }^{1, *}$ \\ 'Samsung Medical Center, Seoul, Korea (yooyoung.lee@samsung.com) ${ }^{2}$ Sungkyunkwan University School of Medicine, Seoul, Korea}

Objective: The aim of this study is to investigate the role of conization prior to radical hysterectomy in patients with early cervical cancer.

Methods: Patients who had radical hysterectomy for early cervical cancer at Samsung medical center from 2001 to 2020 were eligible. Patients were divided into 2 groups; patients with pre-operative conization or patients who had biopsy.

Results: A total of 1,826 patients were included; 294 patients with pre-operative conization (16.1\%, group 1$)$, and 1,532 patients without conization (83.9\%, group 2). 255 patients in group 1 showed resection margin positives after conizations. Patients in group 1 were younger ( 46 years vs. 48 years, $\mathrm{p}<0.001)$. Non-squamous histology $(29.9 \%$ vs. $22.8 \%, \mathrm{p}=0.014)$ and bulky cervical mass (>4 cm, $16.9 \%$ vs. $4.1 \%$, p<0.001) were more frequent in group 2. Laparotomy was more popular in group 2 (70.5\% vs. $51.4 \%, \mathrm{p}<0.001)$, the proportion of type of radical hysterectomy was similar (type $3,98.2 \%$ vs. $97.6 \%$, $\mathrm{p}=0.472)$. On final pathology after radical hysterectomy, larger tumor size, depth of invasion more than $1 / 2$, lymphovascular space invasion positive, pelvis lymph node (LN) positive, para-aortic LN positive, microscopic parametrial invasion were more frequently observed in group 2 . When adjusting these factors in Cox model, patients with pre-operative conization showed survival benefit in progression free survival (hazard ratio $[\mathrm{HR}]=0.709 ; 95 \%$ confidence interval $[\mathrm{CI}]=0.480-1.045$; $\mathrm{p}=0.082$ ) and in overall survival (OS; HR $=0.449 ; 95 \% \mathrm{CI}=0.276-$ $0.278 ; \mathrm{p}<0.001)$.

Conclusion: Our data suggest the therapeutic role of conization before radical hysterectomy in early cervical cancer which showed better OS.

Oral (OC6)

Cervical Cancer

https://doi.org/10.3802/jgo.2021.32.S1.0c6

\section{Definitive radiotherapy for cervical adenocarcinoma: outcomes and immune- related factors}

\author{
Yuhei Miyasaka,' Yuya Yoshimoto, ${ }^{2, *}$ Kazutoshi Murata, ${ }^{3}$ \\ Ken Ando, ${ }^{4}$ Takeshi Ebara, ${ }^{5}$ Noriyuki Okonogi, ${ }^{3}$ Hayato Ikota, ${ }^{6}$ \\ Hideaki Yokoo, ${ }^{7}$ Tatsuya Ohno, ${ }^{4}$ Takashi Nakano ${ }^{8}$ \\ 'Gunma University Heavy Ion Medical Center, Maebashi, Japan \\ ${ }^{2}$ Department of Radiation Oncology, Fukushima Medical University, \\ Fukushima, Japan (yyoshimo@fmu.ac.jp) \\ ${ }^{3}$ QST Hospital, National Institutes for Quantum and Radiological Science \\ and Technology, Chiba, Japan \\ ${ }^{4}$ Department of Radiation Oncology, Gunma University Graduate School \\ of Medicine, Maebashi, Japan \\ ${ }^{5}$ Department of Radiation Oncology, Kyorin University, Tokyo, Japan \\ ${ }^{6}$ Department of Diagnostic Pathology, Gunma University Hospital, \\ Maebashi, Japan \\ ${ }^{7}$ Department of Human Pathology, Gunma University Graduate School of \\ Medicine, Maebashi, Japan \\ ${ }^{8}$ National Institute for Quantum and Radiological Science and Technology, \\ Chiba, Japan
}

Objective: Data on prognosis and immune-related factors specific for cervical adenocarcinoma (CA) treated with definitive radiotherapy (RT) were insufficient. To evaluate these, we conducted a multi-institutional retrospective analysis.

Methods: Patients with CA treated with definitive RT comprising external beam RT and intracavitary brachytherapy were included. Pretreatment biopsy samples were stained with programmed death-ligand 1 (PD-L1) and CD8 antibodies. Associations of these molecules with clinical outcomes were evaluated.

Results: A total of 71 patients were included in this study. Median follow-up period was 37 months (range, 5-194). 
Platinum-based chemotherapy was concurrently administered for $44(62 \%)$ patients. 3/5-year overall survival (OS) rate and progression-free survival (PFS) rate were $64 / 50 \%$ and $40 / 37 \%$, respectively. Membranous expression of PD-L1 was positive in $8.5 \%$ of patients, and not associated with prognosis.

The presence of tumor-infiltrating $\mathrm{T}$ cell positive for CD8 (CD8+TILs) in the tumor nest was positive in $83 \%$ of patients, and associated with favorable OS $(\mathrm{p}=0.037)$ but not with PFS $(p=0.52)$. A Cox regression analysis showed that the presence of CD8+TILs was an independent predictor of favorable OS (hazard ratio $=0.19 ; 95 \%$ confidence interval $=0.065-0.57$ ) as well as lower International Federation of Gynecology and Obstetrics stage (2008, I-II vs. III-IVA), maximum tumor diameter smaller than $40 \mathrm{~mm}$, and use of concurrent chemotherapy.

Conclusion: We reported clinical outcomes of CA after definitive $\mathrm{RT}$ and expression of immune-related molecules using biopsy sample. Our findings suggested that CD8+TILs in the tumor nest was a potential predictor of favorable prognosis, although prognostic impact of membranous PD-L1 expression was unclear.

Oral (OC7)

Preinvasive Disease of Cervix, Vulva, and Vagina

https://doi.org/10.3802/jgo.2021.32.S1.0C7

\section{Prognostic outcomes and risk factors for recurrence after laser vaporization for cervical intraepithelial neoplasia: a single- center retrospective study}

Keisuke Kodama, ${ }^{1}$ Hideaki Yahata, ${ }^{1, *}$ Kaoru Okugawa, ${ }^{1}$ Hiroshi Yagi, ' Masafumi Yasunaga,' Tatsuhiro Ohgami, Ichiro Onoyama, ${ }^{1}$ Kazuo Asanoma, ${ }^{1}$ Mototsugu Shimokawa, ${ }^{2}$ Kiyoko Kato'

'Kyushu University, Fukuoka, Japan (hyahata@med.kyushu-u.ac.jp) ${ }^{2}$ Yamaguchi University, Yamaguchi, Japan

Objective: Cervical intraepithelial neoplasia (CIN) is a precancerous lesion that may progress to invasive cervical cancer without intervention. We aim to examine the prognostic outcomes and risk factors for recurrence after laser vaporization for CIN 3, CIN 2 with high-risk human papillomavirus (HPV) infection, and CIN 1 persisting for more than 2 years.

Methods: Between 2008 and 2016, a total of 1,070 patients underwent cervical laser vaporization using a carbon dioxide laser. We performed a retrospective review of their medical records to assess their clinical characteristics, pathologic factors, and prognostic outcomes.

Results: The mean patient age was 34 years (range, 18-64 years). The preoperative diagnosis was CIN 1 in 27 patients, CIN 2 in 485 patients, and CIN 3 in 558 patients. Over a median follow-up period of 15 months, the 2-year recurrence rate was $18.9 \%$, and the 5 -year recurrence rate was $46.5 \%$. The 2 -year retreatment rate was $12.6 \%$, and the 5 -year retreatment rate was 30.5\%. We diagnosed 9 patients with invasive cancer after treatment; all patients underwent combined multidisciplinary treatment, and there were no deaths during follow-up. The recurrence-free interval was correlated with patient age (hazard ratio $[\mathrm{HR}]=1.028$; 95\% confidence interval $[\mathrm{CI}]=1.005-1.051$; $\mathrm{p}=0.0167)$, body mass index $(\mathrm{HR}=1.052 ; 95 \% \mathrm{CI}=1.008-1.098$; $\mathrm{p}=0.0191)$, and glandular involvement $(\mathrm{HR}=1.962 ; 95 \%$ $\mathrm{CI}=1.353-2.846$; $\mathrm{p}=0.0004$ ).

Conclusion: Cervical laser vaporization is effective and useful for patients with CIN who wish to preserve fertility. However, patients with glandular involvement, older age, and higher body weight require close follow-up for recurrence.

\section{Uterine corpus cancer}

Oral (OM1)

Endometrial Hyperplasia, Endometrial Intra-epithelial Neoplasia, and Endometrial Cancer

https://doi.org/10.3802/jgo.2021.32.S1.0M1

\section{The real-world experience of pembrolizumab and lenvatinib in recurrent endometrial cancer: a multicenter study in South Korea}

\footnotetext{
Junhwan Kim, ${ }^{1}$ Joseph J. Noh, ${ }^{2}$ Tae Kyoung Lee, ${ }^{3}$ Se Ik Kim, Jung-Yun Lee, ${ }^{3}$ Jeong-Won Lee, ${ }^{2}$ Jae-Weon Kim ${ }^{1, *}$

'Department of Obstetrics and Gynecology, Seoul National University College of Medicine, Seoul, Korea (kjwksh@snu.ac.kr)

${ }^{2}$ Samsung Medical Center, Sungkyunkwan University School of Medicine, Seoul, Korea

${ }^{3}$ Department of Obstetrics and Gynecology, Women's Cancer Center, Institute of Women's Life Medical Science, Yonsei University College of Medicine, Seoul, Korea
}

Objective: To investigate real-world experience of pembrolizumab and lenvatinib (PEMBRO+LEN) in patients with recurrent endometrial cancer in South Korea.

Methods: This retrospective cohort study included patients with recurrent endometrial cancer who received PEMBRO+LEN from January 2020 to May 2021 in three tertiary hospitals in South Korea. We summarized patient characteristics and evaluated response rates, survival outcomes, and treatment-related adverse events (AEs).

Results: In total, 48 patients were included in the study. The median age was 62.5 (42-78) years. The most common histologic subtype was endometrioid $(43.8 \%)$ followed by serous (25.0\%). Most patients were MMR proficient (91.7\%). Programmed death-ligand 1 and P53 status were positive 